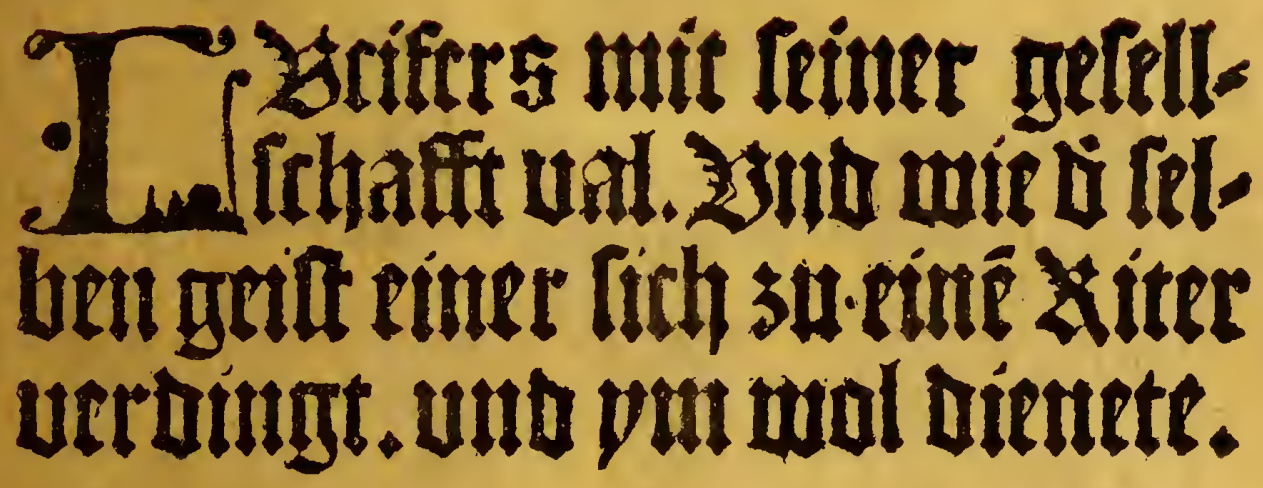


Digitized by the Internet Archive in 2015

https://archive.org/details/lucifersmitseine00unse 


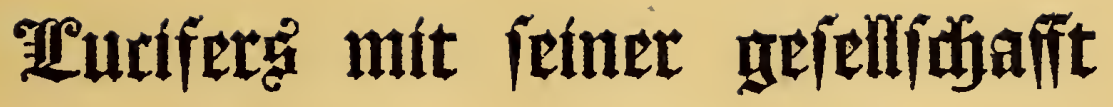

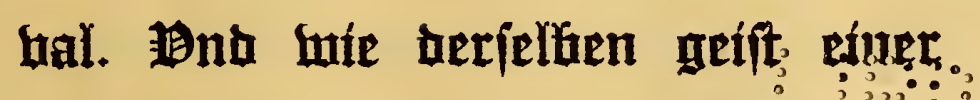

fidf $3 \mathfrak{u}$ einem Mitter berdinint

unt um mal tienet:

25 amferg 1493

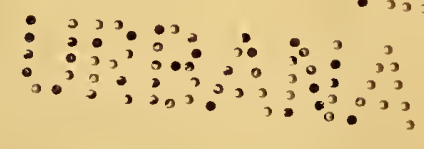

t

Nach dem Unicum

im

\section{Germanischen National-Museum}

\author{
za NÜRNBERG
}

in Facsimile herausgegeben

t

FRANKFURT a. M.

JOSEPH BAER \& Co.

1895 


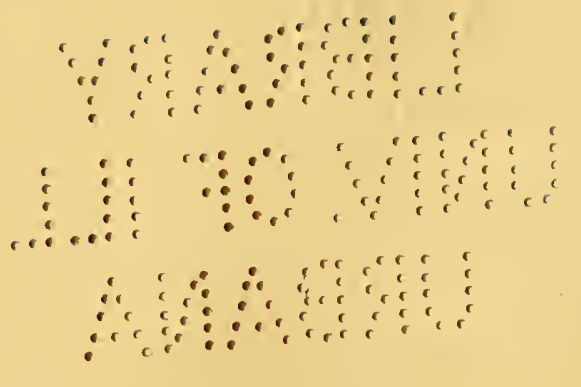

In too Exemplaren ausgegeben 
F. LC. 0 von Lucifers mit seiner gesellschafft val uin der Schwarzischen Sammlung in Altdorf”. G. W. Panzer hat es in den "Annalen der ältern deutschen Litteratur" I 372 registrirt, offenbar ohne es selbst gesehen zu haben, denn er gibt gegen seine Gewohnheit keine Collation an. Seine Angaben sind wiederholt und erörtert worden in einer stattlichen Reihe von Schriften, deren Liste K. Engel aufgestellt hat (Zusammenstellung der Faustschriften, Oldenb. 1885 . Anhang Nr. r.), aber niemand hat das Buch selbst gesehen oder Bestimmtes über seinen Inhalt erfahren. Die Schwarzsche Sammlung ist verschollen, sie soll nach England verkauft worden sein (P. Schwenke, Adressbuch d. D. Bibliotheken 1893, I16), und ein zweites Exemplar von Lucifers val war in keiner öffentlichen Bibliothek, in keiner Privatsammlung zu finden.

Ein glücklicher Zufall hat uns nun zur Entdeckung eines Exemplars dieses verlorenen Buches geführt. Wir haben es in unserem Antiquarischen Anzeiger 444

125956 
S. $30 \mathrm{f}$. angekündigt, und die Vermuthung, die wir dabei aussprachen, dass es ein Unicum sei, ist uns seitdem von verschiedenen Seiten bestätigt worden. Das Germanische National-Museum in Nürn$\therefore$ b.e r:g : ladi das: Schriftchen von uns erworben, sodass $\because$ es: đơn á̉ átschen Gelehrtenwelt gesichert ist. Die : zahtreicher a Áfragén, die bei dieser Gelegenheit an $\ldots$...ins gerichtet wurden, haben uns bestimmt die $\mathrm{Be}-$ nützựg gós Originals durch vorliegendes Facsimile :zí" élèrchtérn;"das wir mit Erlaubniss des Germanischen National-Museums veröffentlichen.

Frankfurt a. Main, im November 1895 .

\section{JOSEPH BAER \& CO.}




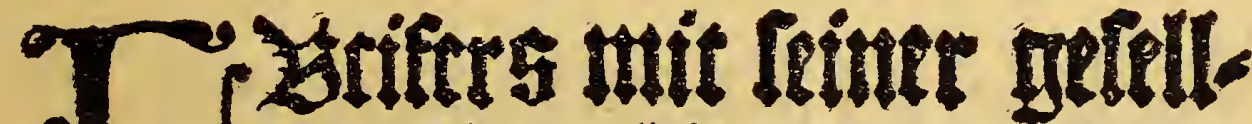

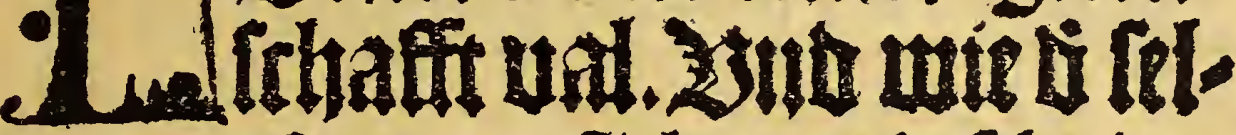

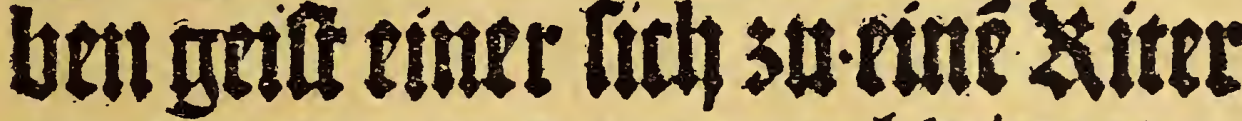 utrungt. unt pat wol tientets.}

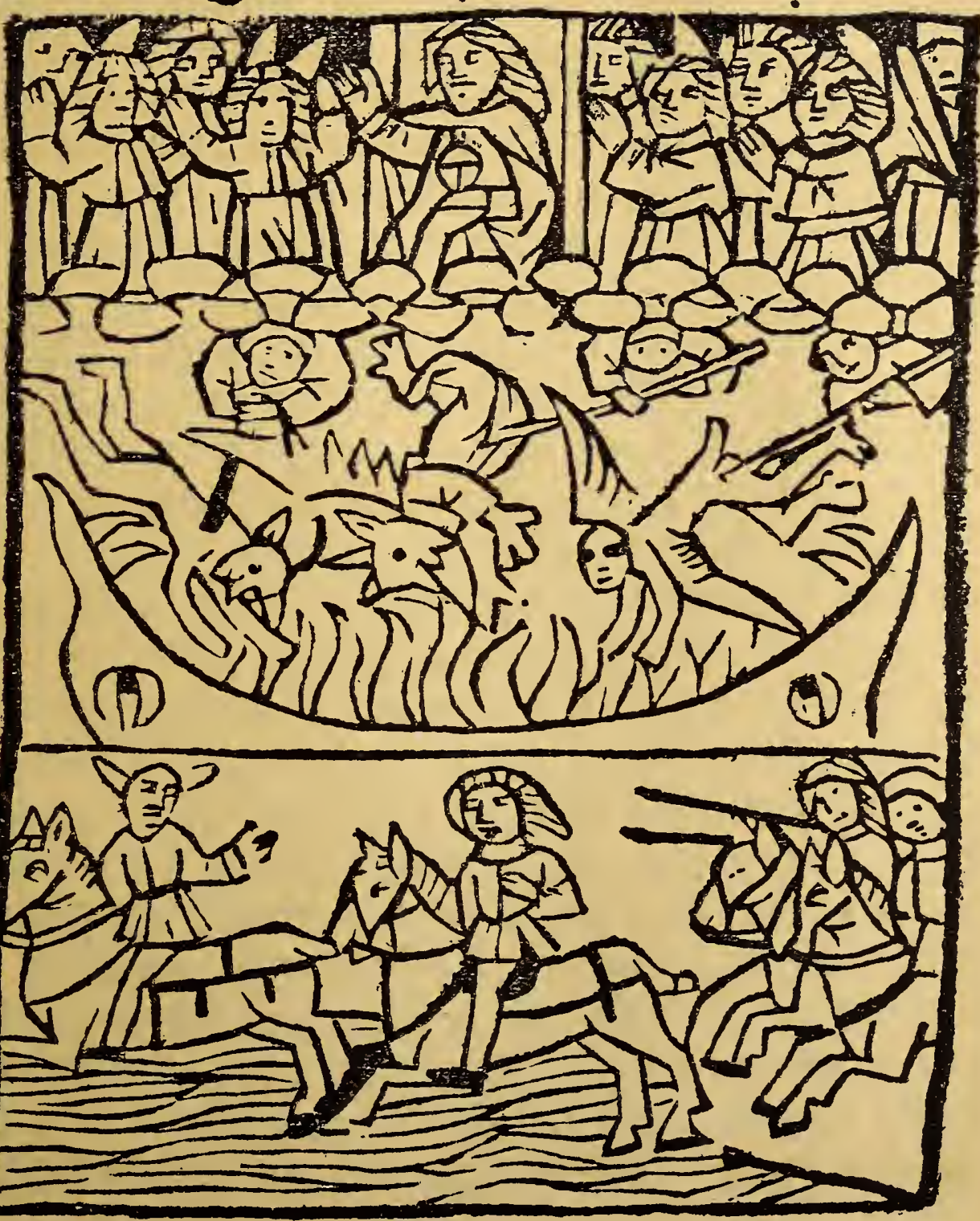




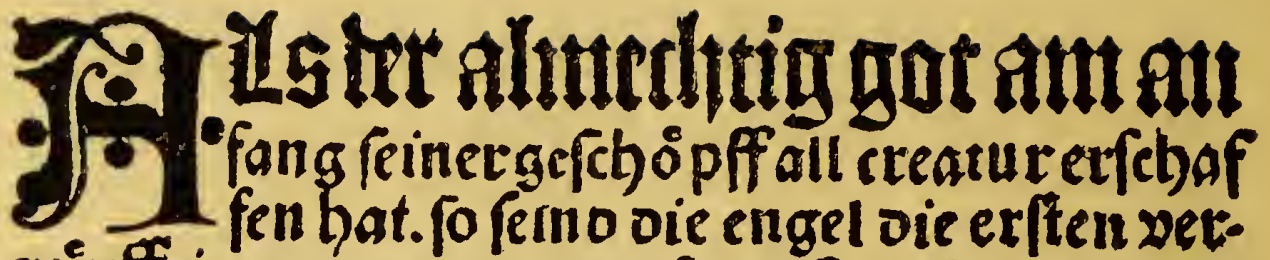
minffrigen creatur getwe (en. vin nach oernatur gar eoel loblich yñ witoig. wñ wnroen all mit sinanoer erfehaffen. Tno als oie heiligen leter meinent fo ift oas gechehen in oe wout als got fprach fiat lur.oas ift. cs foll weroen bas liecht als gefchriben feet in oem bueb oer gefel) ópffe am erften capitel. Zber fy wuroen nit in gleich

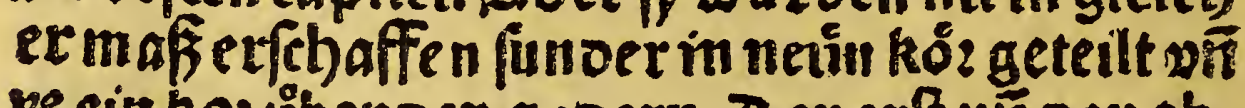
ve cin koz uber oen andern. Ber erft bin oer ob. enf koz ift genant Seraphin. Derander 5 he rubin.oerozit Ihzonus. oer viert $B$ ominatio nes. Derfinffi pincipatus. ver fech) potefta ter. oerfibeut Dirtures. oer acht Zatehangeli. per neint Tingeli. vno bat vetlicher ko? fein be funoere wuteckung wĩ ambt von got sé herten etlich fieno alsoeg vo2 oé angeficht gotes pñ lo ben ynoeren got in prinenoer on ynauffprech enlicber liebe. etlicb vallent nioer auffire an . líis fo fy got loben vi eten.etlich feino oen men fchen zugeol oent oas is is befehirmen wi beht. ren yoz oen pófen geifté yii oas tono ín mit grof fem cenftlichem vléis. vñ die oberften engel ge. pietent yno verkúnoen ben ynoern oen willen gotes. pin bat ein yetlicher engel oarnach frecio kunft vno wilfen nach oe yno er got clerlicher feben xï anfebaupen mag oañ die aberften en 


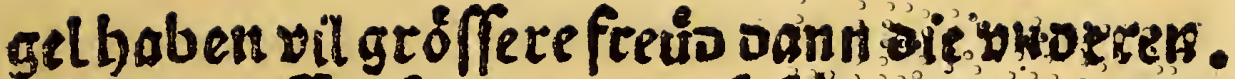
anch fo willen fy mer oanin fy feberi on mittet in oen fpiegel oer heiligen oriualrigkeit.jar in alle kunftono wiffen ift. ynd nllengel wuroen yon exfen er[chaffen oas fy irnferien boillets ge baben mochren. TI Ger oen was ciner gar eoel yno kóftich ưber oie andern oer fich oali feiner zieto yno kơfticheit úberbub vno meint et woolt got geleich frin. pin [prach al fo die wort oie vns feheeibt22 [ayas in oem.xiii. capitel. 7ch wito auffeigen úber oie bóbe derwoleken yñ wiro geleich oein oberffen. Dno was allfo fein will ono meinung oas ym oie anoern engel fol ten onoerrenig vügebor fam fein pno ymere et pieten oie allein gott 34 geb)ozt. Zu/o warent vil engel oie ym folicber eren gunoen yno ym a! fo feinerboffart veruolgtê. oocheiner mer oañ oer anver. Don ftunoan fraffet got die felben boffart als 2 [ayas fchreibt iu oem genanten ca

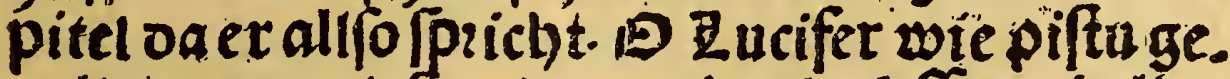
uallen sno wirft getzogen in oie tieff oer belle. Dno da waro er alfo mit feiner aefelf chaffit anis geftoffen oon oere wigen freío yno wato oair frewo verwanoelt in oie ewigen pein yno mare, ter. Dino als 7Ricolans oe $\#$ yia (chicibt (auch anoer lecer) fo feino von yegklichem koz enlicts engel geualten.aber einer in grófere pein oañ ber anoer oarnach yno ym yegklicher oer eren ge ginnt bat vno got fein lob ynoere entzieben wolt wan etlicbengel fino geuallen in oie tieff 
aexhellentititem berten oafy ymer ynd ewigk litheit multen beleiben. etlich in oen lutffit xno

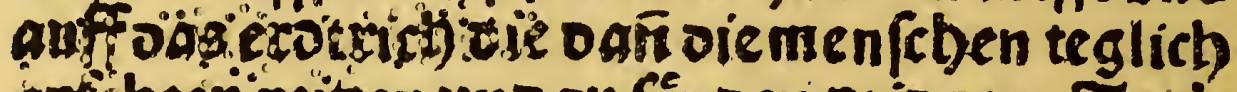
anjechen reitsen pno zu fínden pringen. Zuuch To fehrestbi sturctus zaguftinus im puch von aen. Iriiï. fragen oas etlich engel oie oer bof. fart pno poß̧beit nit gant, verwilligen gefallen feino in oen lufft pno oie felben beleivigen oen menfshen nit. funoerfy beftetigen die menfebe in gntem fürfás. Dñif etlicher lecer meinnng Das die felben engel am les)(ten gerich)t gnao er werben muigen gen oem almechtigen got. ooch ob oas fey ooer nit fey oas weciß niemano. oamn gottee gericht heimlich mono verborgen feino. yna was er in oen oder moern oingen fut wer. willigt bno verbengt oss if alles wol gethon. vno nir on gros mercklich we fach) Des heimlich ent gericht gottes.

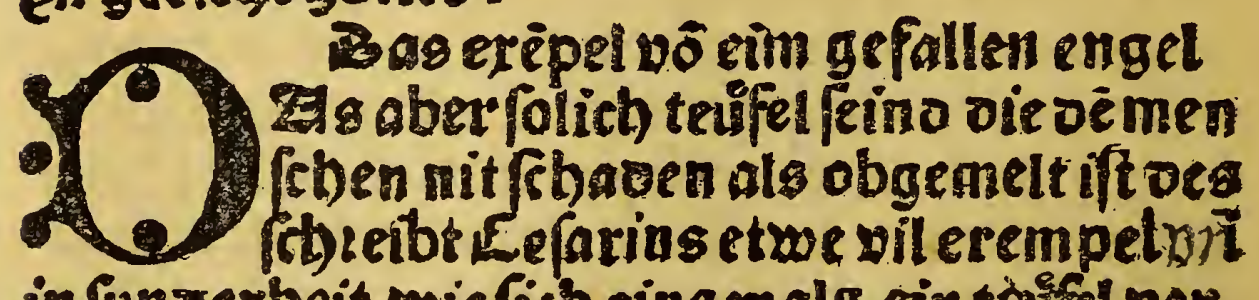
in funverbeit wie ficb rins mals ein tèripl pet, soanoelt in ein geftalt eins wol getziecten iưng ling8 ono kam 341 einé ritter ono wato fein dis ner.oer waro oé ritter gar genem ví gar wol. geuallen, yno er vieneym garmitgroffen oleis pmo tresulichen, pno fo mit ftólichen geperoen. oas fich oer ritter aes yerwonndert. Zer rireet far noch nie auffein pfero noch orkab ex a 
Allweg gegensourtig yī pogfeine knié xli hiele ym ore flegreiff. Ha un fügtes fich oas ly eins tag es mit cinans riten bin kamen an ein gros 20 af. fer oa eilten dem ritter fein feino nact) die yn be getten yinb fein leben zubzingen. Ba (prach 6 ritter zu oem knecht. Woir imuiflen fterben Daî mein feino eilen yns nach fo migen wir nit úb er Dag woafferkumen. fo kinden woit auch funft niendert bin flieben. Ba jpzach o knecht. beer ir folt oes nit vózchten ich weí Be on furt wol in oenus waffer wolgt mir nach fo wo ollen wir wo ol entrínnen. Ba fpzach oer ritter es wer nie ge. boit wozeen oas ein furt ourch oas wafler we re. Doch reiter ó knecht riach) vii kain all fo on allen febaoen oureh oas waffer. Din oas oes rit. ters peino auch an oas wafferkamen oa heten [y gros verwundern wie o ritter úber oas wo of

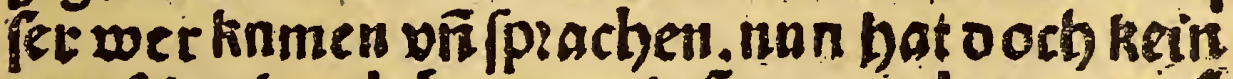
menfch nic gehózt oas rin futt ourch oas waf. Fer gee viñ porcheren yn prin wolten ym nicht tmee nach yolgen. IB arnach fingt es fich oas oes rit. ters haulf caw gar krầck toaco pisinuffoen to De oas all ertit am ir wertzwe eifelten. oa prach $\delta$ kneche 3 um berren if fach oas mā fo beftreiche mit ciner ló win milch fo poirt fy gefuno. Bert ter(prach wie mag mâfy aber habe, Er (prach. icts wil [ gar palo bingen. pno ee oain rn eine. ftnno. vinals palo Rom er vii beftreich fy osmit vion ftuno soaro fy gefind. Ber ritier fragt pon toann er fy prachlyete. Ee [pzach aus zarabia 
wö einer fetigenden lơw in oic hab ich gemolkkē Baerfebzack oer ritter von difen wozten und fragt yn wer er wer. Er antwozt ym vö fpzàch Bufolt on alle forg fein ich pin oein knecht vî boil oir tretilich) Dienen on allen 3 we ifel. $3 a \mathrm{ab}$ ex Der ritter ye woilfen wolt werer wer. oa fpr.

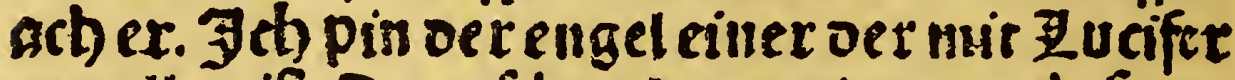
gellallen iff. Ba erfflbzack Der ritter noch ferer vno pipach. Go ounnnein teúfel pift wie haft ou mir fo getreullich migen oienen. Bafprach oer telffel. Eo ift mir gar eill geofler troft oas ich bey oen menfechen wonen fol win ich pit oich Das ou mich behalteft 3 neinem knecht ich will jir on allen 3 weifel gang treúlich dienen yî fol Dirkein fchaa nimmermer von mix gefebeben. $27 b e r$ oer ritter wolt fein nit mer 34 einem kine che oper viener. pno fragt yn was ex ym $3 a$ lon geben folt oas wolt er gern thon dann er here

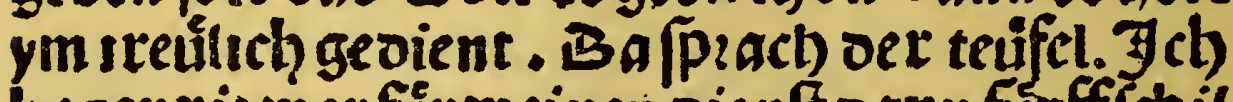
beger nit mer fír meinen oienf toann funffich il ling für oie kanffe ein glócklein wno hengket fy in vie capellen oas man fy an oen funtagen leưtr oas oie gelaubigen menfchen oaroureb gefam melt weroen 3 oem góllichen oienft. Dno als er olfer wozt (ptach) oa ver (chwander er oen an gen oes ritters. CT Zu us oifemel épel mag man gar wol mercken oas etlich teiffel pine (als ZAu guftínus fébreibt) oic den mêf(t)en nit (ch)aden. funoer fy 3 guten wereké siben. Zaber ob fy aun lerften gngo erwerben oocr nit oas ift viemant 
swiften. yno diefelbẽ engel haben gar wenig ge. willigr in oie fino

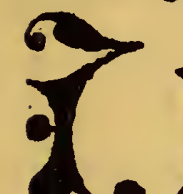

Tho ox alfo oer wal oer engel gefebach - vilifi verwandelt wu coen in teuffel on iwuraen oie andern engel oie dem iza cifer folicher boffart nie ge williget herren allfa beftetiget ourch oie genao gottes oas fy ewigk lichen fir bas luimernier gefún den múgell, Dat umb oas fy fich $\delta$ góclichen maye fart von ftano an nach item erfehaffen oemúrigklichen ynoer tenig pno gebor (än machten. Diro als Tbylari us fichee ibruber $A$ battheum fo veroienen iy teg lichen vno wirr ir feligkeit gemert fo fy die men fichen befchirmen yus oem górlichen millen ya oertenig machen. Thierumb wato 1 Hcifer mit feiner gefelfchaffe alfo ecthoze in iter hoffart viz poßßtheic aus [ye wigklichen mimermer kein ge. noo erwerben múgell pno begern. sno wirt ic pein yii marter téglich gemerc oaî iy teglichen finnoén fo fip oie menfeberin anfechten vno $3 u$ (tind en reizen. Dno wie wol ir pein oarumb geme ret wirt fo fino fy ooch fo neioig oas fy oie pein gern leíen nur oas oie menfchen mit yn verto ren yno oer groffen fretioe oar aus ingeffolfen feino beraube weroen. Zl fo fehzelbr Ilicolans oe Zyza úber oas puch oer ge(chóp ff oagefehii benf fet von oem val oes erfitumen/chen. Bes gleichen auch amoer lerer.

É etruckt 3u 25amberg yon meyfter

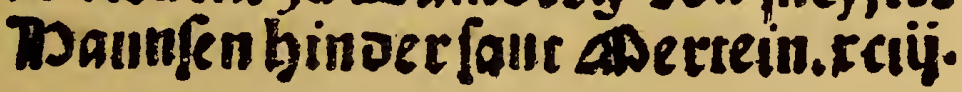




UNIVERSTYY OF ILLINOIS-UABANA

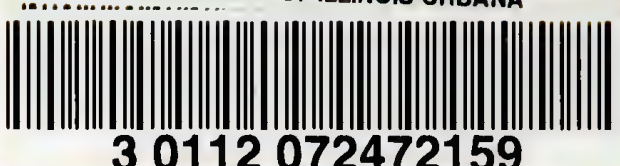

30112072472159 